EPJ Web of Conferences 59, 07001 (2013)

DOI: $10.1051 /$ epjconf/20135907001

(C) Owned by the authors, published by EDP Sciences, 2013

\title{
Overview of PETAL, the multi-Petawatt project in the LMJ facility
}

\author{
N. Blanchot ${ }^{1, a}$, G. Behar ${ }^{1}$, T. Berthier ${ }^{1}$, B. Busserole ${ }^{1}$, C. Chappuis ${ }^{1}$, \\ C. Damiens-Dupont ${ }^{1}$, P. Garcia ${ }^{1}$, F. Granet ${ }^{1}$, C. Grosset-Grange ${ }^{1}$, \\ J.-P. Goossens ${ }^{1}$, L. Hilsz ${ }^{1}$, F. Laborde ${ }^{1}$, T. Lacombe ${ }^{1}$, F. Laniesse ${ }^{1}$, \\ E. Lavastre ${ }^{1}$, J. Luce ${ }^{1}$, F. Macias ${ }^{1}$, E. Mazataud ${ }^{1}$, J.L. Miquel ${ }^{2}$, J. Néauport ${ }^{1}$, \\ S. Noailles ${ }^{1}$, P. Patelli ${ }^{1}$, E. Perrot-Minot ${ }^{1}$, C. Present ${ }^{1}$, D. Raffestin ${ }^{1}$, B. Remy ${ }^{1}$, \\ C. Rouyer ${ }^{1}$ and D. Valla ${ }^{1}$
}

1 CEA, DAM, CESTA, 15 avenue des Sablières, CS 60001, 33116 Le Barp Cedex, France

2 CEA, DAM, DIF, 91297 Arpajon, France

\begin{abstract}
The status of the PETAL project is presented in this paper. The global architecture and performances of this facility are detailed with the first experimental results obtained on the LIL facility, and with the main steps which will allow shooting in the center of the target chamber. Some technical issues like wavefront correction, damage threshold in femtosecond regime and focusing are presented.
\end{abstract}

\section{INTRODUCTION}

Transferring PETAL (PETawatt Aquitaine Laser) from the LIL (LMJ prototype) to the LMJ building (Figure 1) was decided in 2010. The PETAL project consists in the addition of one short-pulse (500 fs to $10 \mathrm{ps)}$ ultra-high-power, high-energy beam (few kJ compressed energy) to the LMJ facility. PETAL will offer a combination of a very high intensity multi-petawatt beam, synchronized with the nanosecond beams of the LMJ. PETAL will be used for High Energy Density Physics and research on Fast Ignition, as a first step for the HiPER European project.

The PETAL project is divided in 5 phases. The $1^{\text {st }}$ one, finished in 2008 , concerned the key issues (front end [1] and compression [2,3]). The $2^{\text {nd }}$ and $3^{\text {rd }}$ phases concern the installation of the front end, amplifier section, and the compression stage, the $4^{\text {th }}$ phase, the transport-focusing stage in the target bay before the end of 2014. The $5^{\text {th }}$ phase will be dedicated to the coupling of PETAL with LMJ.

\section{FIRST EXPERIMENTAL RESULTS}

First, we design a specific front end for PETAL [1]. The challenge is to amplify femtosecond and large spectrum pulses $(100 \mathrm{fs} / 16 \mathrm{~nm})$ up to energy of $100 \mathrm{~mJ}$ without nonlinear effects (B-integral $\sim 0 \mathrm{rad}$ ). At this stage, a beam shaper is used to adapt the beam profile to the amplifier section, which has been designed for square beams.

An Öffner stretcher creates $9 \mathrm{~ns}$ chirped pulse duration, starting from 100 fs @ $16 \mathrm{~nm}$ pulse. The last step is the pre-amplification stage. This stretched pulse is then amplified in a two stages OPCPA

\footnotetext{
ae-mail: blanchot@projet-lmj.org
}

This is an Open Access article distributed under the terms of the Creative Commons Attribution License 2.0, which permits unrestricted use, distribution, and reproduction in any medium, provided the original work is properly cited. 


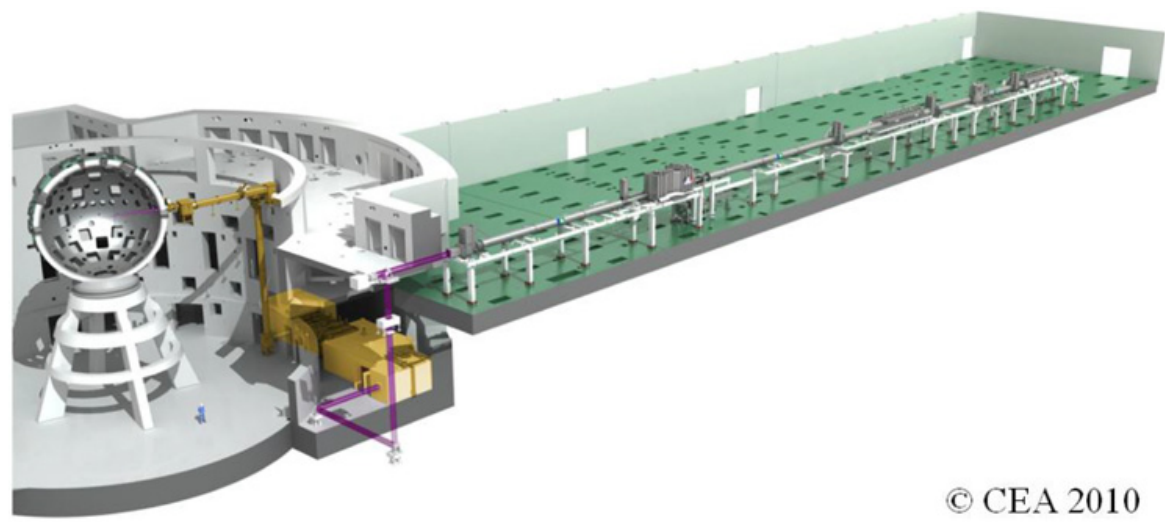

Figure 1. PETAL in the LMJ Building.
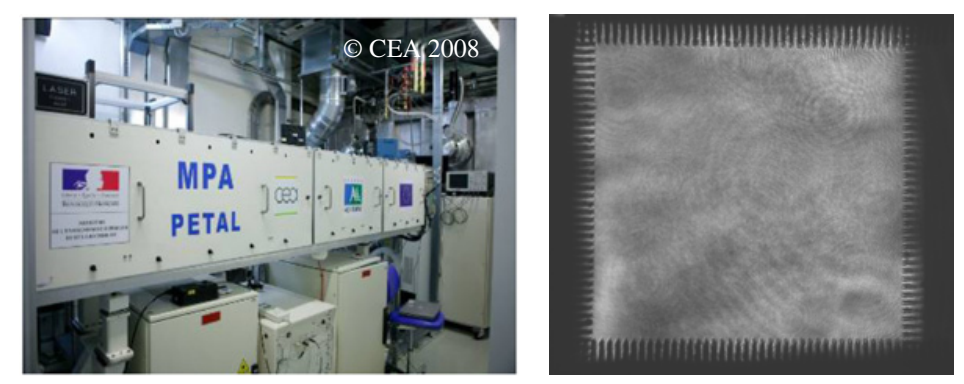

Figure 2. PETAL pre-amplifier module in the LIL facility and beam profile after OPCPA stage.

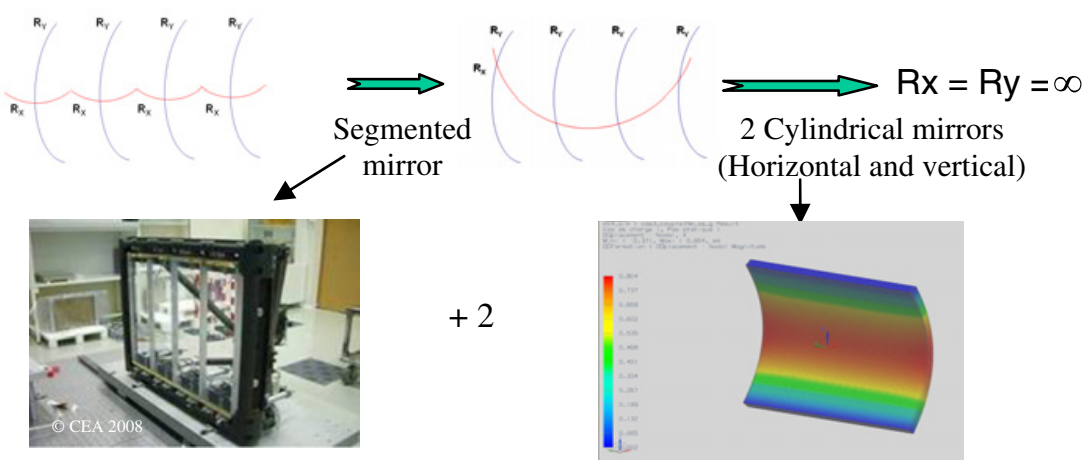

Figure 3. Principle of the compressor wavefront correction.

configuration (Optical Parametric Chirped Pulse Amplifier) integrated in a PAM box (figure 2, left view). The first stage consists of two LBO crystals of $25 \mathrm{~mm}$ length; the second stage of one BBO crystal of $12 \mathrm{~mm}$ length. This configuration delivers 100 to $120 \mathrm{~mJ}$ signal energy with a spatial contrast uniformity of $12 \%$ on the full aperture (figure 2, right view). The pump beam energy is in the range of $800 \mathrm{~mJ}$ at $532 \mathrm{~nm}$ with $4.5 \mathrm{~ns}$ duration. Tilts of crystals were carried out to avoid parametric fluorescence and the BBO crystal length was adjusted to optimize the energy extraction of the second stage. 


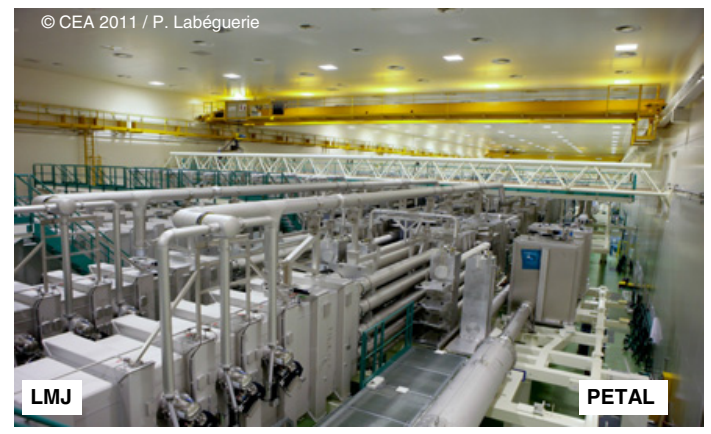

Figure 4. View of the amplifier section of PETAL.

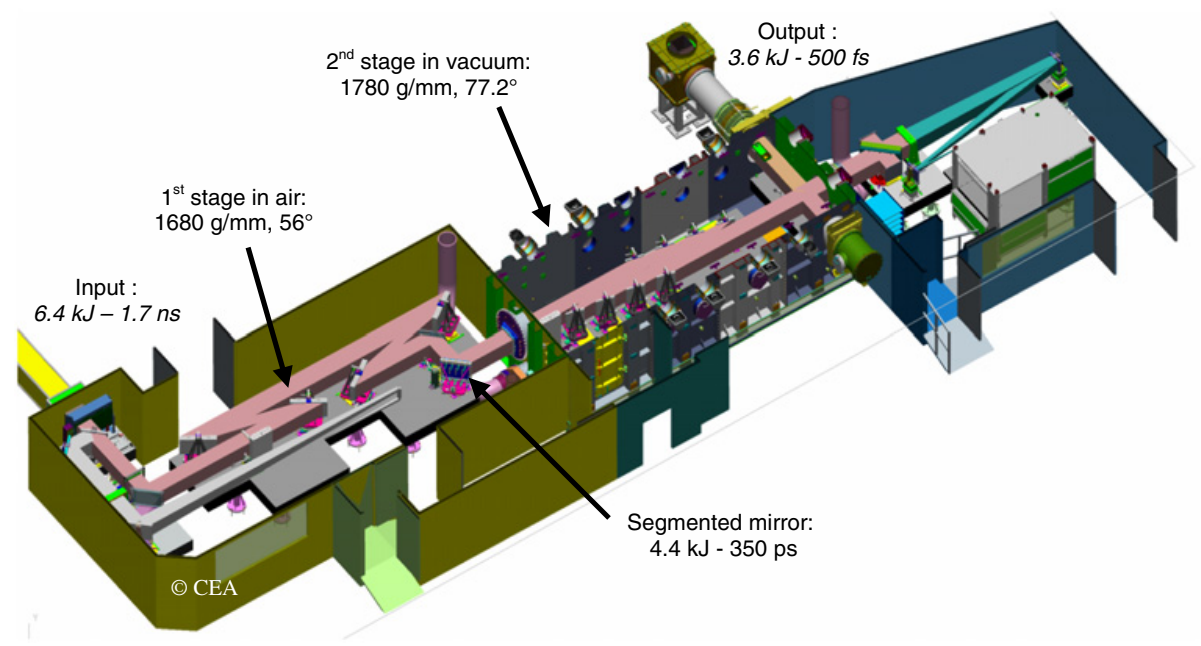

Figure 5. Subaperture compression scheme: 2 stages with 4 independent compressors in vacuum.

Concerning thecompression stage, the subaperture compression scheme has been retained. Experimental demonstrations [2] of compression (650 fs), synchronisation of the 4 independent compressors ( $<50 \mathrm{fs})$ and beam phasing $(50 \mathrm{~nm}$ piston, $1 \mu \mathrm{rad}$ tilts) have been demonstrated using a segmented mirror and specific diagnostics (extension of the SID4 [3] and 2D-spectral interferometry [2]).

Damage test on gratings has shown that $4 \mathrm{~J} / \mathrm{cm}^{2}$ @ $500 \mathrm{fs}$ can be reached for a $77.2^{\circ}$ angle of incidence [4]. During this campaign, transport mirrors were tested. Due to their lowest incident angle $\left(45^{\circ}\right)$, the maximum damage threshold can not exceed $2.5 \mathrm{~J} / \mathrm{cm}^{2}$. Studies are under progress to overcome this limitation but for the moment, the maximum energy compressed by PETAL will be limited to $1 \mathrm{~kJ}$.

The first experiments on the compression stage have also shown that the optical surface of the grating is modified when the compression vessel is under vacuum, due to the extraction of water in the multi-layer coating. In our case, as for segmented gratings, this creates at the output of the compressor, a wavefront equivalent to a corrugated iron. To correct this wavefront deformation, we will use the segmented mirror in a first step to generate a beam with pure astigmatism (figure 3 ) and then we are developing two cylindrical mirrors to compensate the two different curves in the horizontal and vertical directions. 


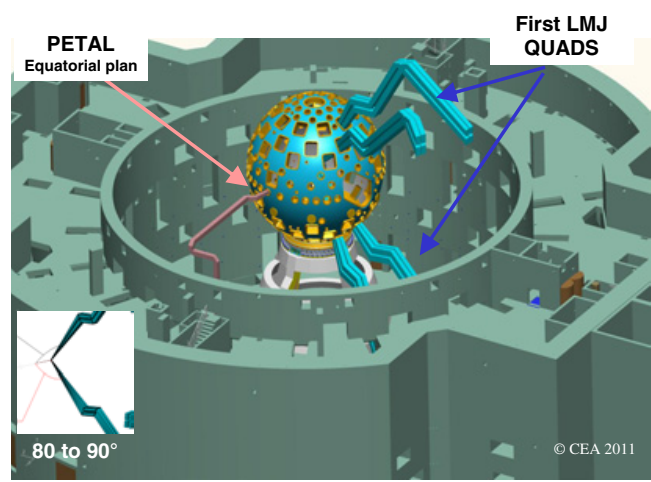

Figure 6. Beam arrivals in the target chamber.

\section{INSTALLATION IN THE LMJ BUILDING}

The installation of PETAL in the LMJ building has started in 2010. The infrastructures of the amplifier section are installed (figure 4).

The compression scheme (figure 5) is similar to the scheme designed for the LIL facility [2], improvements have been done to limit the spectral cut-off of the compressor. The main adaptations concern the electromagnetic compatibility and the nuclear reservation.

After compression, the beam is propagated under vacuum by 5 mirrors. A reservation has been taken during mechanical conception to adapt in a future the $2 \omega$ conversion of the beam for contrast enhancement or to adapt the polar of the beam.

Concerning the focalisation, an off axis parabola ( $90^{\circ}$ deviation, $7.8 \mathrm{~m}$ focal length) followed by a pointing mirror is used to focus the beam into target. The exploration of the center of the target chamber is $+/-50 \mathrm{~mm}$, by adjustment of the pointing mirror or displacement of both the parabola and pointing mirror for focusing. The alignment of the parabola is provided by a reference mirror representing the parent parabola. Mechanical reservations are made on the pointing mirror to install in the future 2 mirrors to realize 2 beams on target.

Figure 6 shows the arrivals of the firsts LMJ quads and the PETAL beam in the target chamber. The angles between PETAL and the quads are between 80 to $90^{\circ}$.

This work is being performed under the auspices of the Conseil Régional d'Aquitaine, of the French Ministry of Research and of the European Union and with the scientific supports of the Institut Lasers et Plasmas.

\section{References}

[1] E. Hugonnot, G. Deschaseaux, O. Hartmann, H. Coïc, Appl. Opt. 46, 8181 (2007)

[2] N. Blanchot, E. Bar, G. Behar, C. Bellet, D. Bigourd, F. Boubault, C. Chappuis, H. Coïc, C. Damiens Dupont, O. Flour, O. Hartmann, L. Hilsz, E. Hugonnot, E. Lavastre, J. Luce, E. Mazataud, J. Neauport, S. Noailles, B. Remy, F. Sautarel, M. Sautet, C. Rouyer, Opt. Express 18 (10), 10088 (2010)

[3] S. Mousset, C. Rouyer, G. Marre, N. Blanchot, S. Montant, and B. Wattellier, Opt. Lett. 31, 2634 (2006)

[4] J. Neauport, E. Lavastre, G. Razé, G. Dupuy, N. Bonod, M. Balas, G. de Villele, J. Flamand, S. Kaladgew, F. Desserouer, Opt. Express 15, 12508 (2007) 